\title{
Lipid Core Plaque Distribution Using Near-infrared Spectroscopy Is Consistent with Pathological Evaluation in Carotid Artery Plaques
}

\author{
Masashi KotsugI, ${ }^{1}$ Ichiro NAKAGaWA, ${ }^{1}$ Kinta HataKeyama, ${ }^{2}$ \\ HunSoo PARK, ${ }^{1}$ Fumiya SATO, ${ }^{1}$ Takanori FURUTA, ${ }^{1}$ Fumihiko NisHIMURA, ${ }^{1}$ \\ Shuichi YAMADA, ${ }^{1}$ Yasushi MOTOYAMA, ${ }^{1}$ Young-Soo PARK, ${ }^{1}$ \\ and Hiroyuki NAKASE ${ }^{1}$ \\ ${ }^{1}$ Department of Neurosurgery, Nara Medical University, Kashihara, Nara, Japan \\ ${ }^{2}$ Department of Pathology, Nara Medical University, Kashihara, Nara, Japan
}

\begin{abstract}
Carotid artery stenting (CAS) is performed as a treatment for carotid artery stenosis. However, lipid-rich plaques cause embolic complications and sequelae. Near-infrared spectroscopy (NIRS) can identify lipid components by applying a near-infrared absorption pattern, and the distribution of lipid components can be evaluated as the maximum lipid core burden index (maxLCBI). Intravascular ultrasound (IVUS) equipped with NIRS has been clinically applied recently, and its diagnostic usefulness and validation have been reported for coronary arteries; however, its consistency with actual pathological diagnosis in carotid artery lesions has not been validated. In this study, we investigated the consistency between the maxLCBI values and histopathological diagnoses. Patients with cervical carotid artery stenosis who underwent carotid endarterectomy (CEA) were examined in this prospective study. Pathological diagnosis was determined after NIRS evaluation, which was performed on the extracted plaques ex vivo. The histological slices of decalcified and paraffin-embedded sections were stained by hematoxylin-eosin (HE) and Elastica van Gieson (EVG), and for low-density lipoprotein (LDL), C-reactive protein (CRP), CD68, and glycophorin $\mathrm{A}$. The correlation between maxLCBI values and histological findings. Seventy lesions assessed by NIRS were pathologically analyzed. There was a positive linear correlation between maxLCBI values and pathological findings as determined by HE (angle), HE (area\%), EVG, CRP, and CD68 staining (respectively, $r=0.624, p<0.001 ; r=0.578, p<0.001 ; r=0.534$, $p<0.001 ; r=0.723, p<0.001 ; r=0.653, p<0.001)$. In conclusion, the maxLCBI values assessed by NIRS showed a significant positive linear correlation with pathological evaluations in carotid lesions. The maxLCBI values in carotid arteries are consistent with pathological evaluations.
\end{abstract}

Keywords: imaging modalities, carotid artery stenosis, lipid plaque, NIRS-IVUS, carotid artery stenting

\section{Introduction}

As endovascular techniques and devices have advanced, carotid artery stenting (CAS) has become an alternative to carotid endarterectomy (CEA) for carotid artery stenosis. However, accurately evaluating preoperative plaque characteristics are essential

Received May 13, 2020; Accepted June 25, 2020

Copyright $\subseteq 2020$ by The Japan Neurosurgical Society This work is licensed under a Creative Commons AttributionNonCommercial-NoDerivatives International License. because lesions with lipid-rich plaques put patients at risk of complications leading to sequelae in CAS. ${ }^{1,2)}$ Plaque protrusions (PP) appear in the lumen of stents after stenting in patients with lipid-rich carotid plaques, and PP is strongly correlated with perioperative ischemic complications. ${ }^{1)}$ Evaluation of plaque characteristics using magnetic resonance imaging (MRI) is commonly used to assess carotid plaques. ${ }^{3-}$ 5) Recent quantitative color-coded T1-weighted MR plaque imaging for preoperative plaque diagnosis can accurately distinguish lipid core and intraplaque hemorrhages (IPHs) ${ }^{5}$; however, high-resolution MRI is not always an option for patients before 
revascularization. ${ }^{6}$ An additional imaging modality that can accurately identify the lipid components of carotid plaque at high risk for embolic complications during CAS would be therefore required.

Near-infrared spectroscopy (NIRS) is a diagnostic imaging technique that can identify lipid components by applying a near-infrared absorption pattern that is different from that of other tissues. ${ }^{7}$ Recently, an intravascular diagnostic imaging device using NIRS, NIRS-intravascular ultrasound (NIRS-IVUS), has been clinically applied. NIRS can accurately evaluate lipid core plaque (LCP) distributions, and a correlation between NIRS diagnosis and clinical prognosis in coronary artery lesions has been demonstrated. ${ }^{8)}$ Furthermore, a consistent relationship between NIRS evaluation and pathological diagnosis in coronary artery plaques has been found. According to comparisons with pathological coronary tissue in autopsy cases, the sensitivity of lipid plaque detection on NIRS is $90 \%$ and the specificity is $93 \%{ }^{9}{ }^{9}$ However, the consistency with actual pathological plaque diagnosis in carotid artery lesions has not been studied. The purpose of the present study was to investigate the consistency between evaluations of the lipid core distribution using NIRS and pathological findings for carotid plaque specimens.

\section{Methods}

\section{Study population}

From April 2018 to February 2020, consecutive patients with cervical carotid artery stenosis who underwent CEA were examined in this prospective study. The subjects were cases with carotid artery stenosis over 20 years old who underwent CEA for carotid stenosis with surgical indications according to the Japanese Guidelines for the Management of Stroke 2015 (Supplement 2019). All examinations in this study were performed after obtaining approval from the institutional review board (No. 1936) and written informed consent from each patient.

\section{CEA procedure}

All CEAs were performed under general anesthesia. A skin incision was performed at the forward edge of the sternocleidomastoid muscle to expose the common carotid artery, external carotid artery, and internal carotid artery for dissection around connecting tissues. The carotid plaque was carefully removed in a one-piece fashion without cutting inside through a fibrous cap (Fig. 1). For carotid plaque evaluation using NIRS-IVUS, the extracted specimens were excised without making an incision in the thickened intima and the lumen was kept intact to avoid plaque damage. After removal of the specimen, the

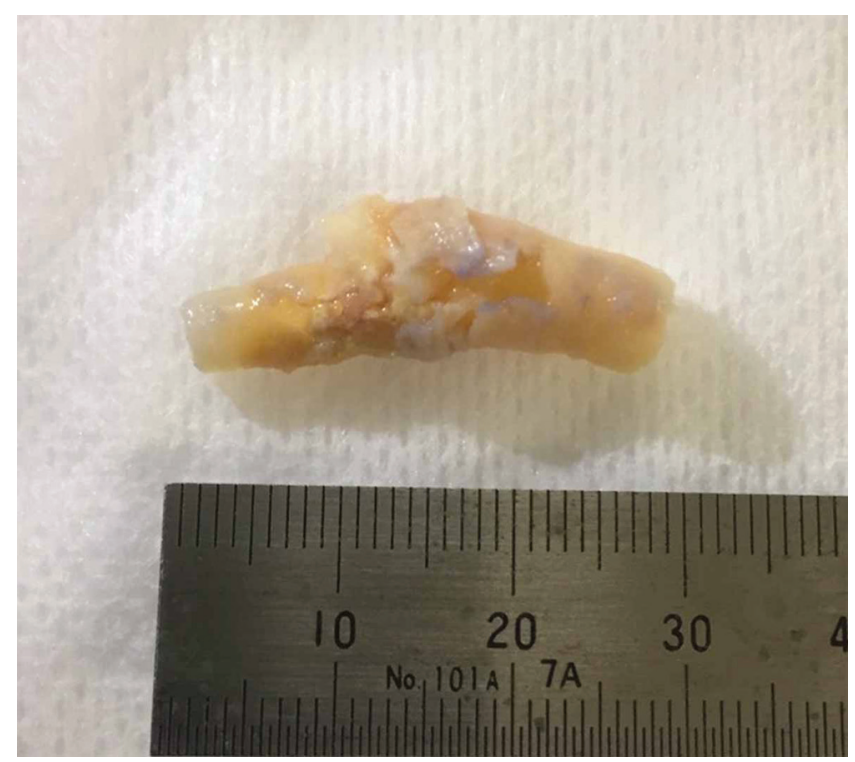

Fig. 1 The extracted carotid plaque in a one-piece fashion.

incised carotid artery was sutured water-tightly with 3-0 GORE-TEX suture (W. L. Gore \& Associates, Inc., Newark, DE, USA), and the operation was completed with meticulous hemostasis.

\section{NIRS-IVUS procedure and maxLCBI analysis}

Ex vivo NIRS-IVUS assessment of excised carotid plaque specimen was subsequently performed. First, the wrap was removed from the intimal plaque of the carotid artery with gauze and immersed in a container filled with saline. Second, a 3.2 F Infraredx NIRS catheter (InfraReDx, Burlington, MA, USA) was advanced along a 0.014-inch guidewire (GT wire; Terumo Medical, NJ, USA) through the plaque in saline. The catheter was connected to the TVC Imaging System (Makoto ${ }^{\mathrm{TM}}$; Infraredx), and the LCP was measured by automatic pullback at $0.5 \mathrm{~mm} / \mathrm{s}$. The proximal edge of the specimens was labeled as $0 \mathrm{~mm}$ and associated with the proximal histology thin section. To provide a quantitative summary metric of the LCP over the entire scanned segment, the maximum lipid core burden index (maxLCBI) was calculated. The chemogram displayed color-coded blocks according to the probability of finding a lipid plaque, if the percentage probability value for lipids was $\geq 0.98$, the block showed yellow. LCBI is the fraction of LCPpositive area in the chemogram multiplied by 1000 for any $4 \mathrm{~mm}$ segment. The maxLCBI was defined as the maximum value of the LCBI for any $4 \mathrm{~mm}$ segment. The maxLCBI was calculated based on the scanned segment every $4 \mathrm{~mm}$ including the minimal lumen area. After the measurement of the maxLCBI values, the specimen was pathologically evaluated. 


\section{Histological evaluation}

Specimens excised from the carotid artery during CEA were provided for histological evaluation. After fixation by formaldehyde, each segment was cut perpendicular from the proximal side of each $5-\mathrm{mm}$ interval of the plaque. The histological sections of decalcified and paraffin-embedded tissue were stained by hematoxylin-eosin (HE) and Elastica van Gieson (EVG). Serial sections were assessed by immunohistochemical stain using primary antibodies for oxidized low-density lipoprotein (LDL) (rabbit polyclonal antibody, Immundiagnostik AG, Bensheim, Germany), C-reactive protein (CRP) (clone CRP-8, Sigma-Aldrich, St. Louis, MO, USA), CD68 (clone PG-M1, Dako, Glostrup, Denmark), and glycophorin A (clone JC159, DakoCytomation, Carpinteria, CA, USA) using Envision methods as previously described. ${ }^{10)}$ Histopathological features of the plaque were analyzed based on the agreement of a pathologist $(\mathrm{KH})$ who was blinded to the NIVUS and NIRS findings. The proportion of the lipid area was measured using NanoZoomer Digital Pathology ver. 2.7.43 (Hamamatsu Photonics K.K., Hamamatsu, Japan) for all sections stained by HE and EVG. In immunohistochemical staining sections, the ratio of the positive part to the total area was measured. In addition, to investigate the lipid distribution, the angle of lipid distribution from the center of the lumen was measured (Fig. 2).

\section{Data and statistical analysis}

All statistical analyses were performed with SPSS version 20.0 (IBM Corp., Armonk, NY, USA). The correlations between histological specimens and maxLCBI values were analyzed using the Spearman test.

\section{Results}

In all, 15 patients (14 men, 1 woman; age range 65-84 years [mean age 73.5 years]; 11 symptomatic cases; stenosis rate (NASCET ${ }^{11)}$ method), $50 \%$ to $95 \%$ [mean $78.8 \%$ ]) had a vessel assessed by NIRSIVUS, resulting in 70 lesions being analyzed. Clinical characteristics of the patients included symptomatic in 11 patients, hypertension in 10 patients, and hyperlipidemia in 10. In two cases who gave up stenting due to high value of maxLCBI, in vivo NIRS assessment was obtained. maxLCBI values were completely consistent between in vivo and ex vivo NIRS assessment in these cases. Figure 3 shows the representative case. An 84-year-old male with symptomatic carotid artery stenosis underwent CEA. Figure 3 shows the chemogram image of NIRS in vivo (Figs. $3 \mathrm{~A}-3 \mathrm{C}$ ) and ex vivo (Figs. 3D-3F),

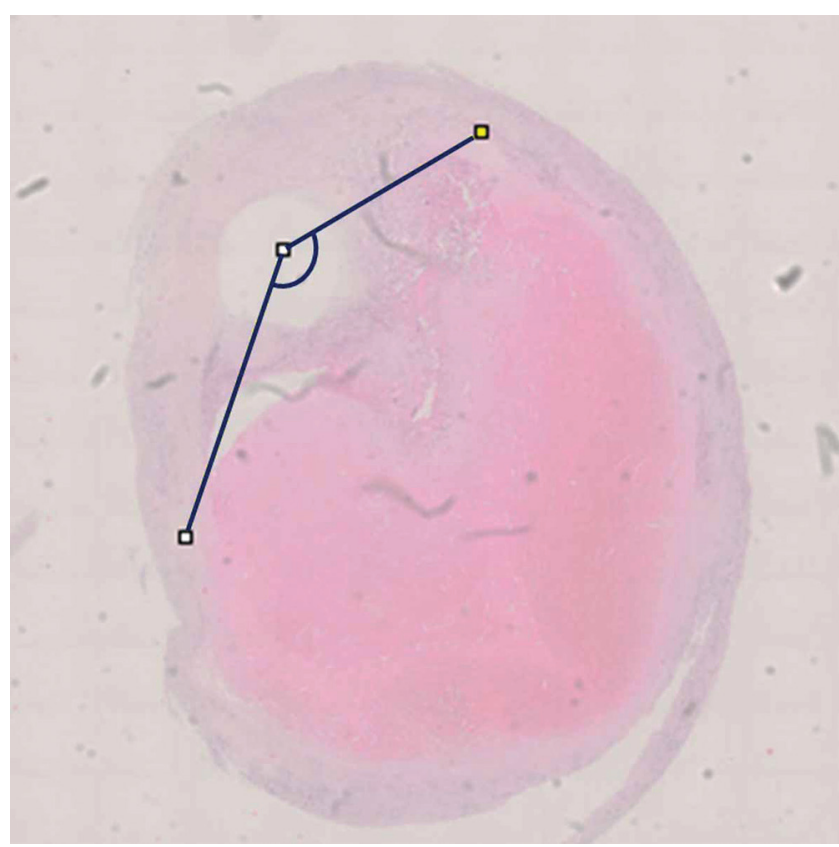

Fig. 2 The angle of lipid distribution was measured from the center of the lumen.

indicating similar maxLCBI value. Figure 4 shows the histological specimens with each stain, the proportion of the lipid area.

Table 1 shows that relationship between maxLCBI and histologic findings using the Spearman test. There was a positive linear correlation between maxLCBI values and lipid angle $(\mathrm{r}=0.624 ; p<0.001)$ (Fig. 5A) and between maxLCBI and lipid area (r $=0.578 ; p<0.001$ ) (Fig. 5B) stained by HE. Furthermore, there was a positive linear correlation between maxLCBI values and lipid areas stained by EVG ( $\mathrm{r}$ $=0.534 ; p<0.001$ ) (Fig. 5C). In immunohistochemical staining evaluation, the percentage areas stained for LDL, glycophorin A, CRP, and CD68 were 15.0 $\pm 16.0 \%, 10.1 \pm 15.7 \%, 16.0 \pm 22.2 \%$, and $18.8 \pm$ $19.0 \%$, respectively. There was a significant positive linear correlation between maxLCBI values and lipid areas stained for CRP $(\mathrm{r}=0.723 ; p<0.001)$ and CD68 ( $\mathrm{r}=0.653 ; p<0.001)$. There was no significant correlation between maxLCBI and lipid areas stained for LDL and glycophorin A.

\section{Discussion}

It has been reported that lipid-rich carotid plaques are prone to ischemic complications after CAS., ${ }^{2,12)}$. Currently, plaque imaging with MRI is commonly performed for preoperative carotid plaque diagnosis; however, accurate evaluation of lipid components of carotid plaques has not been established. In the present study, we evaluated the lipid distribution 

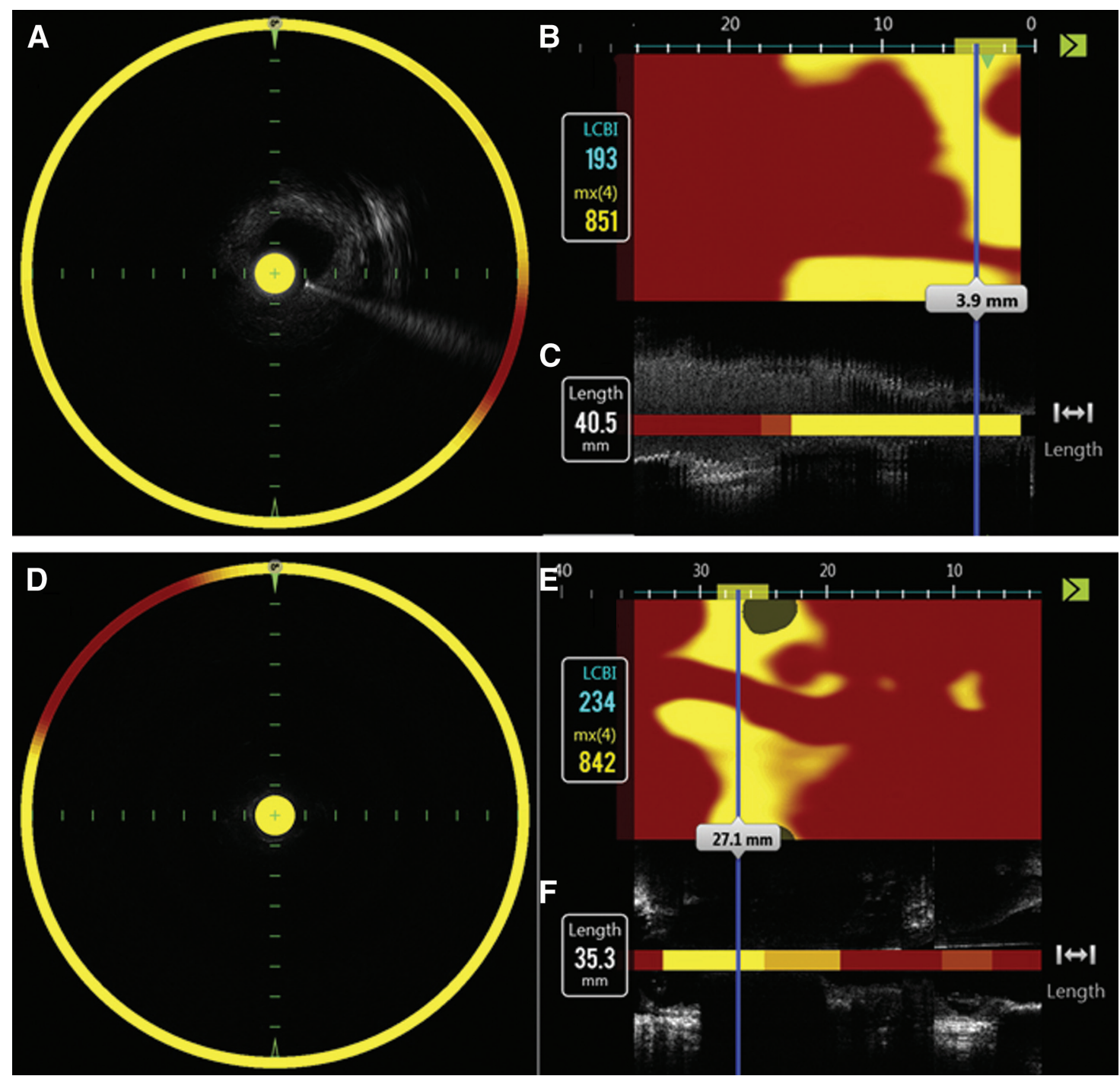

Fig. 3 Representative case of carotid artery stenosis assessed by NIRS scan and histological specimens. A and D indicate fusion image of blood vessel short-axis cross-sectional image and NIRS information by Grayscale-IVUS. $B$ and $E$ indicate chemogram displayed color-coded blocks according to the probability of finding a lipid plaque, if the percentage probability value for lipids was $\geq \mathbf{0 . 9 8}$. $\mathrm{C}$ and $\mathrm{F}$ indicate long-axis cross-sectional image of blood vessel and block chemogram by Grayscale-IVUS. $\operatorname{mx}(4)$ indicates maxLCBI defined as the maximum value of the LCBI for $4 \mathrm{~mm}$ segment of blue bar. Chemogram images of NIRS in vivo (A-C; upper) and ex vivo (D-F; lower), indicating similar maxLCBI value before and after CAE. CAE: carotid endoarterectomy, IVUS: intravascular ultrasound, maxLCBI: maximum lipid core burden index, NIRS: Near-infrared spectroscopy.

of carotid plaques using NIRS-IVUS and the pathological lipid distribution using carotid intima pathological specimens harvested by CEA. We found that the carotid LCP expressed as maxLCBI values was consistent with the pathological evaluations.

In conventional carotid plaque evaluation, several previous studies have compared findings from MR plaque imaging with those from histological evaluation. Advancements in MRI plaque imaging have made it possible to identify high-risk carotid plaques ${ }^{13)}$. Quantitative analysis of high-contrast T1-weighted images can accurately evaluate the composition of carotid plaques according to recent studies. ${ }^{4,14)}$ Previous pathological studies have shown that high intensity lesions on time-of-flight magnetic resonance angiography (MRA) are indicative of IPHs. ${ }^{15,16)}$ IPHs show higher signal intensities than LCP in MR plaque imaging; however, it can be difficult to distinguish LCP distribution where LCP is mixed with IPHs because MR signal intensity of LCP can affect IPHs. ${ }^{3,5,17)}$

NIRS is an imaging technology that can specifically distinguish lipids and has the potential to 

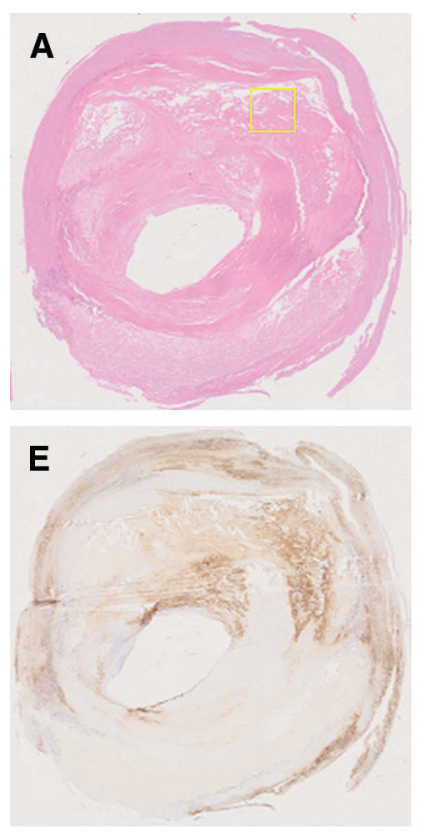
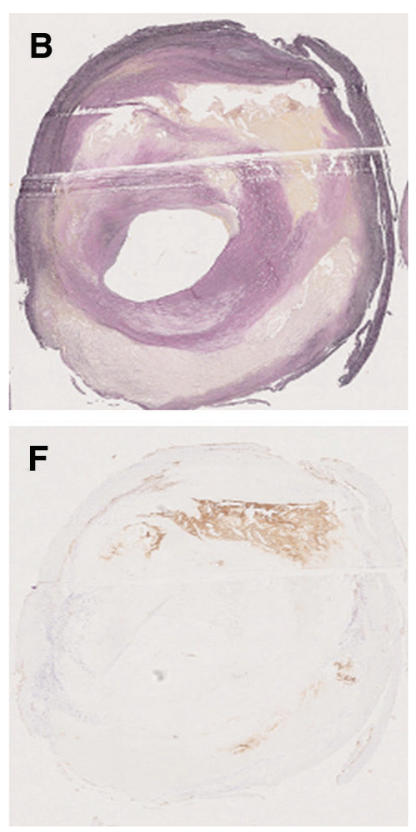
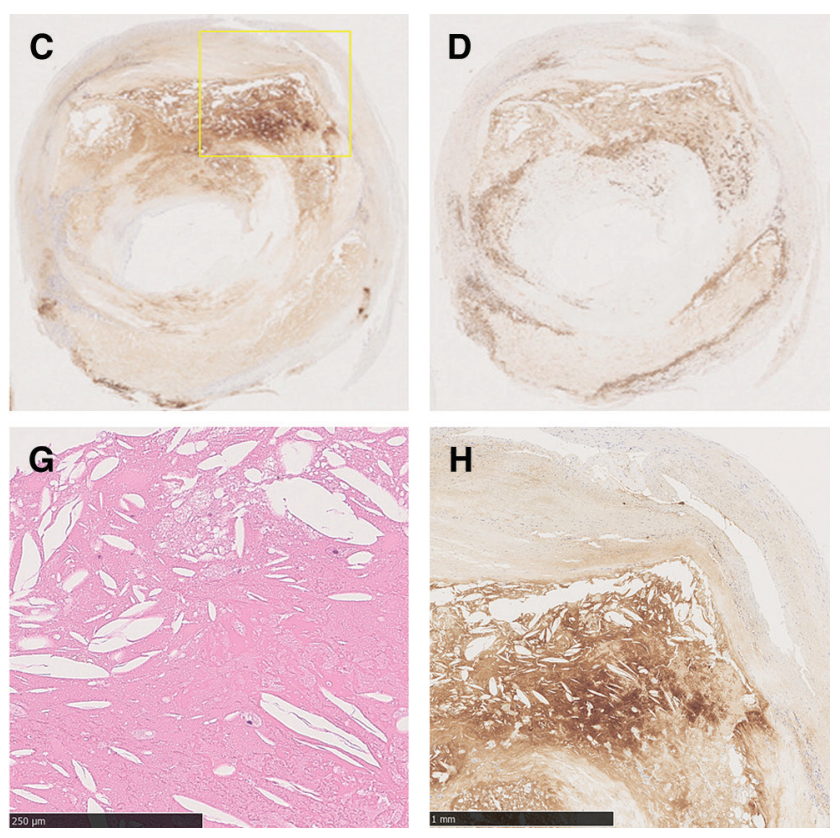

Fig. 4 Example of pathological evaluation. Histological specimens with each staining HE (A), EVG (B), CRP (C), CD 68 (D), LDL (E), and glycophorin A (F). Boxed higher magnification images of HE (G) and CRP (H) show the plaque mainly consist of lipid on microscopic specimens. CRP: C-reactive protein, EVG: Elastica van Gieson, HE: hematoxylin-eosin, LDL: low-density lipoprotein.

Table 1 Correlation between maxLCBI and histologic findings

\begin{tabular}{|c|c|c|c|c|c|c|c|c|}
\hline & & $\begin{array}{c}\text { angle } \\
\left({ }^{\circ}\right)\end{array}$ & $\begin{array}{c}\mathrm{HE} \\
\text { (area\%) }\end{array}$ & $\begin{array}{c}\mathrm{EVG} \\
\text { (area\%) }\end{array}$ & $\begin{array}{c}\text { LDL } \\
\text { (area\%) }\end{array}$ & $\begin{array}{c}\text { CRP } \\
\text { (area\%) }\end{array}$ & $\begin{array}{c}\text { CD68 } \\
\text { (area\%) }\end{array}$ & $\begin{array}{c}\text { Glycophorin } \\
\text { A (area\%) }\end{array}$ \\
\hline \multirow[t]{2}{*}{ maxLCBI } & Correlation coefficient & 0.624 & 0.578 & 0.534 & 0.291 & 0.723 & 0.653 & 0.170 \\
\hline & $p$ Value & $<0.001$ & $<0.001$ & $<0.001$ & 0.015 & $<0.001$ & $<0.001$ & 0.328 \\
\hline
\end{tabular}

CRP: C-reactive protein, EVG: Elastica van Gieson, HE: hematoxylin—eosin, LDL: low-density lipoprotein, maxLCBI: maximum lipid core burden index.

compensate for the shortcomings of current modalities. ${ }^{18)}$ Goldstein et al. revealed that coronary artery lesions with maxLCBI $\geq 500$ are associated with periprocedural myocardial infarction. ${ }^{8)}$ LCP evaluation using NIRS has become a modality to predict outcomes of coronary treatments. ${ }^{19,20)}$. As clinical applications of NIRS for coronary artery diseases, NIRS provides accurate segments with high lipid burden and can select optimal length of the stent. Furthermore, high LCP lesion detected by NIRS can apply filter-type distal embolic protection device during stent deployment avoiding periprocedural myocardial infarction. ${ }^{21)}$ Research for plaque stabilization and reduced complications by stenting and drug therapy for unstable plaques to improve treatment is ongoing. ${ }^{8,22)}$

Although the accuracy of lipid plaque evaluation with NIRS has been suggested for coronary arte ries, ${ }^{8,22,23)}$ there are few reports on the clinical application of carotid artery lesions and therefore no reports on its consistency with histopathological evaluation. ${ }^{24-26)}$ The present study demonstrated a linear correlation between maxLCBI values and pathological evaluations of carotid plaques. We analyzed both the lipid core angle and area in pathological evaluations because the maxLCBI values as measured by NIRS indicate not the area, but the angle of the lipid plaque distribution. Consequently, both the pathological lipid area and angle were correlated with maxLCBI values in this study, which suggests that maxLCBI values indicate carotid lipid core volumes. The high value of maxLCBI which stands for a wide angle of lipid plaque distribution could be a surrogate marker that predicts PP and clinical ischemic events during CAS. 

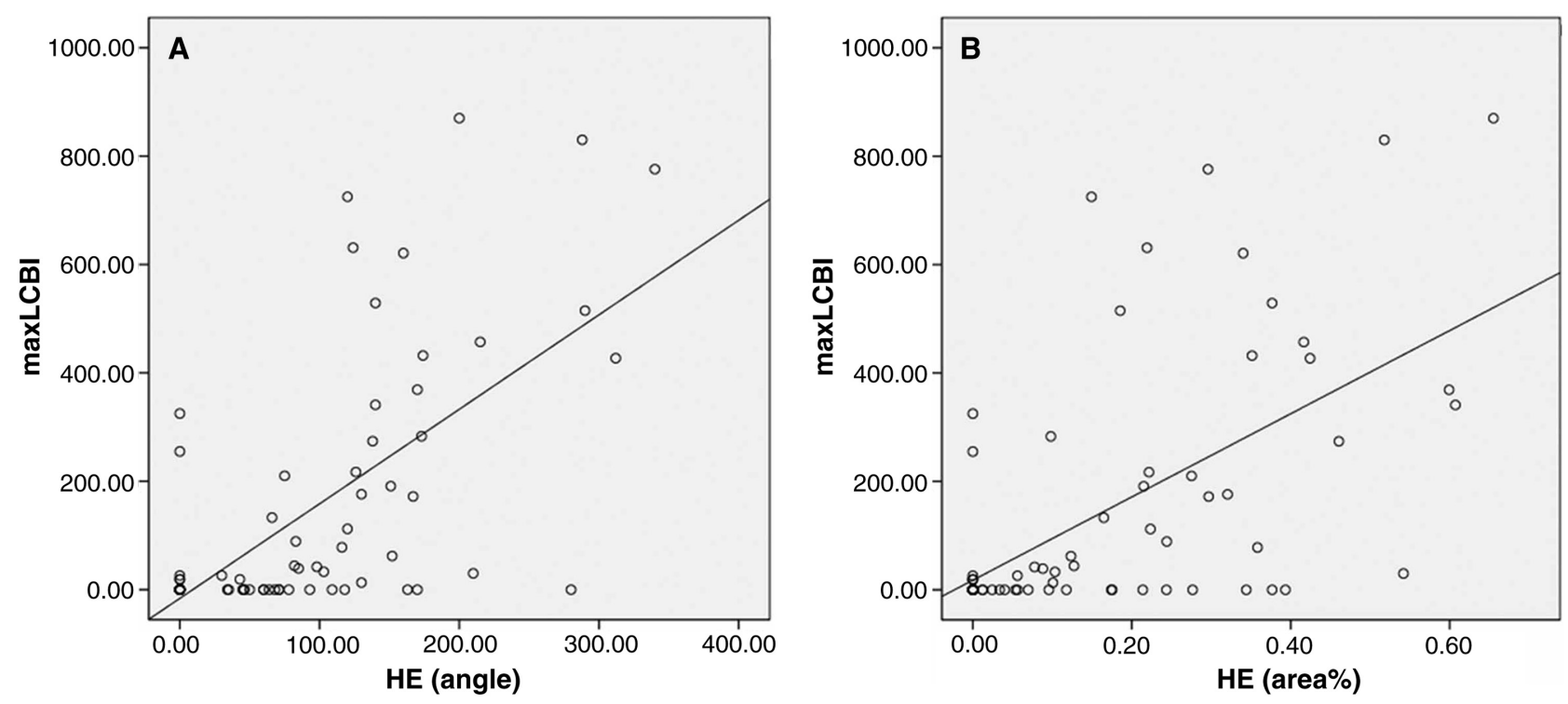

Fig. 5 Relationship between maxLCBI and histologic findings. (A) maxLCBI and angle of lipid core and (B) maxLCBI and area\% stained by HE. HE: hematoxylin-eosin, maxLCBI: maximum lipid core burden index.

Immunohistochemical staining evaluation provides specific information about local inflammation. CRP and CD68 are inflammatory markers. Macrophages form by phagocytosing oxidized LDL cholesterol and remnants deposited on the blood vessel wall, and oxidized LDL, homocysteine, and CRP cause chronic inflammation ${ }^{27,28)}$. The expression of CRP increases in advanced lesions in coronary arteries, and CRP is distributed mainly in LCPs. The expression of CRP reflects a chronic inflammatory state of the vascular wall caused by the deposition of oxidized LDL. In the present study, there was a significant correlation between maxLCBI values and CRP/CD68-positive areas. Some reports have described that the CRP-positive rate is involved in unstable plaques. ${ }^{10,27)}$ The restenosis rate is high in groups with high CRP-positive rates in histological specimens after atherectomy of coronary arteries. ${ }^{27)}$ Furthermore, CD68-expressing macrophages are localized in the lipid cores of unstable plaques without bleeding. ${ }^{10}$ This is consistent with the present results. In contrast, oxidized LDL is considered to be a key factor in the initiation and acceleration of atherosclerosis. The association between oxidized LDL and the instability of carotid plaques has been reported. ${ }^{29)}$ Although there was no correlation between maxLCBI values and the percentage area stained by oxidized LDL in this study, the phase of atherosclerotic development including an acute or chronic stroke phase may have influenced the results. Further study will be required to clarify the association between maxLCBI values and oxidized LDL considering the atherosclerotic phase.
In the present study, we found that NIRS can accurately evaluate the distribution of LCP in carotid plaques, and this was validated by pathological consistency. Greyscale-IVUS is widely used for assessing plaque morphology, and virtual histology IVUS (VH-IVUS) has been developed to improve on the plaque characterization of greyscale-IVUS. However, previous study suggested that VH-IVUS overestimates lipid LCP in the presence of calcification, which can explain the discrepancy between VH-IVUS and NIRS findings in coronary artery study. ${ }^{30)}$ NIRS can provide an additional information to discriminate LCP at high risk for cerebral embolism during CAS. Precise assessment of LCP in carotid plaque with catheter-based NIRS could be clinically applied to embolic protection system modification including flow reversal with Mo.Ma, stent selection such as mesh-covered or double-layered stent during CAS procedure and judgment of additional medication including lipid-modification and antiplatelet therapies. ${ }^{31,32)}$ Recently, Sato et al. found that IPH rather than LCP is more likely to cause embolic complications in CEA procedures. ${ }^{33)}$ Therefore, switching CAS to CEA in patients with vulnerable plaques and high maxLCBI values may help to avoid perioperative embolic complications. Treatment selection based on the maxLCBI values as measured by NIRS could be an important diagnostic modality to improve clinical outcomes in patients who undergo CEA or CAS.

\section{Limitations}

The present study has several limitations. First, the number of cases was small. Second, maxLCBI 
evaluation was performed ex vivo because of the invasiveness of NIRS-IVUS procedure in vivo. The specimens obtained by CEA are intimal plaques, not from the whole blood vessel, which could influence the maxLCBI values. Third, the main limitation of catheter-based NIRS imaging technique is its invasiveness, which precludes its utilization in asymptomatic patients. Finally, the differences in vessel diameter between coronary arteries and carotid arteries may influence the maxLCBI values as measured by NIRS. However, in all the specimens in the present study, we were able to measure the maxLCBI values. Further studies with a larger number of samples are needed.

\section{Conclusions}

maxLCBI values assessed by NIRS showed a significant positive linear correlation with pathological evaluations in carotid plaque. The maxLCBI values in carotid arteries were consistent with pathological evaluations.

\section{Conflicts of Interest Disclosure}

No declaration of sources of funding.

No statement of authorship, conflicts of interest, ethical adherence, and any financial disclosures.

\section{References}

1) Kotsugi M, Takayama K, Myouchin K, et al.: Carotid artery stenting: investigation of plaque protrusion incidence and prognosis. JACC Cardiovasc Interv 10: 824-831, 2017

2) Sakamoto M, Taoka T, Nakagawa H, et al.: Magnetic resonance plaque imaging to predict the occurrence of the slow-flow phenomenon in carotid artery stenting procedures. Neuroradiology 52: 275-283, 2010

3) Cai J, Hatsukami TS, Ferguson MS, et al.: In vivo quantitative measurement of intact fibrous cap and lipid-rich necrotic core size in atherosclerotic carotid plaque: comparison of high-resolution, contrast-enhanced magnetic resonance imaging and histology. Circulation 112: 3437-3444, 2005

4) Narumi S, Sasaki M, Ohba H, et al.: Predicting carotid plaque characteristics using quantitative colorcoded T1-weighted MR plaque imaging: correlation with carotid endarterectomy specimens. AJNR Am J Neuroradiol 35: 766-771, 2014

5) Takaya N, Cai J, Ferguson MS, et al.: Intra- and interreader reproducibility of magnetic resonance imaging for quantifying the lipid-rich necrotic core is improved with gadolinium contrast enhancement. J Magn Reson Imaging 24: 203-210, 2006

6) Chung GH, Jeong JY, Kwak HS, Hwang SB: Associations between cerebral embolism and carotid intraplaque hemorrhage during protected carotid artery stenting. AJNR Am J Neuroradiol 37: 686-691, 2016

7) Caplan JD, Waxman S, Nesto RW, Muller JE: Nearinfrared spectroscopy for the detection of vulnerable coronary artery plaques. J Am Coll Cardiol 47: C92-96, 2006

8) Goldstein JA, Maini B, Dixon SR, et al.: Detection of lipid-core plaques by intracoronary near-infrared spectroscopy identifies high risk of periprocedural myocardial infarction. Circ Cardiovasc Interv 4: 429-437, 2011

9) Moreno PR, Lodder RA, Purushothaman KR, Charash WE, O'Connor WN, Muller JE: Detection of lipid pool, thin fibrous cap, and inflammatory cells in human aortic atherosclerotic plaques by near-infrared spectroscopy. Circulation 105: 923-927, 2002

10) Matsuura Y, Hatakeyama K, Imamura T, et al.: Different distribution of pentraxin 3 and C-reactive protein in coronary atherosclerotic plaques. J Atheroscler Thromb 19: 837-845, 2012

11) North American Symptomatic Carotid Endarterectomy Trial C, Barnett HJM, Taylor DW, et al.: Beneficial effect of carotid endarterectomy in symptomatic patients with high-grade carotid stenosis. $N$ Engl J Med 325: 445-453, 1991

12) Yoshimura S, Yamada K, Kawasaki M, et al.: High-intensity signal on time-of-flight magnetic resonance angiography indicates carotid plaques at high risk for cerebral embolism during stenting. Stroke 42: 31323137, 2011

13) Brinjikji W, Huston J, Rabinstein AA, Kim GM, Lerman A, Lanzino G: Contemporary carotid imaging: from degree of stenosis to plaque vulnerability. J Neurosurg 124: 27-42, 2016

14) Zhao $X$, Underhill HR, Zhao Q, et al.: Discriminating carotid atherosclerotic lesion severity by luminal stenosis and plaque burden: a comparison utilizing high-resolution magnetic resonance imaging at 3.0 Tesla. Stroke 42: 347-353, 2011

15) Wildy KS, Yuan C, Tsuruda JS, et al.: Atherosclerosis of the carotid artery: evaluation by magnetic resonance angiography. J Magn Reson Imaging 6: 726732, 1996

16) Yim YJ, Choe YH, Ko Y, et al.: High signal intensity halo around the carotid artery on maximum intensity projection images of time-of-flight MR angiography: a new sign for intraplaque hemorrhage. J Magn Reson Imaging 27: 1341-1346, 2008

17) Saito A, Sasaki M, Ogasawara K, et al.: Carotid plaque signal differences among four kinds of T1-weighted magnetic resonance imaging techniques: a histopathological correlation study. Neuroradiology 54: 1187-1194, 2012

18) Munemitsu T, Ishii A, Okada E, et al.: Ex vivo assessment of various histological differentiation in human carotid plaque with near-infrared spectroscopy using multiple wavelengths. Neurol Med Chir (Tokyo) 59: 163-171, 2019

19) Madder RD, Goldstein JA, Madden SP, et al.: Detection by near-infrared spectroscopy of large lipid core 
plaques at culprit sites in patients with acute ST-segment elevation myocardial infarction. JACC Cardiovasc Interv 6: 838-846, 2013

20) Kini AS, Baber U, Kovacic JC, et al.: Changes in plaque lipid content after short-term intensive versus standard statin therapy: the YELLOW trial (reduction in yellow plaque by aggressive lipid-lowering therapy). J Am Coll Cardiol 62: 21-29, 2013

21) Kilic ID, Caiazzo G, Fabris E, et al.: Near-infrared spectroscopy-intravascular ultrasound: scientific basis and clinical applications. Eur Heart J Cardiovasc Imaging 16: 1299-1306, 2015

22) Waksman R, Di Mario C, Torguson R, et al.: Identification of patients and plaques vulnerable to future coronary events with near-infrared spectroscopy intravascular ultrasound imaging: a prospective, cohort study. Lancet 394: 1629-1637, 2019

23) Ota H, Magalhaes MA, Torguson R, et al.: The influence of lipid-containing plaque composition assessed by near-infrared spectroscopy on coronary lesion remodelling. Eur Heart J Cardiovasc Imaging 17: 821831, 2016

24) Horvath M, Hajek P, Muller JE, et al.: First-in-man near-infrared spectroscopy proof of lipid-core embolization during carotid artery stenting. Arch Med Sci 12: 915-918, 2016

25) Štěchovský C, Hájek P, Horváth M, Špaček M, Veselka J: Composition of carotid artery stenosis and restenosis: a series of patients assessed with intravascular ultrasound and near-infrared spectroscopy. Int J Cardiol 207: 64-66, 2016

26) Štěchovský C, Hájek P, Horváth M, Špaček M, Veselka J: Near-infrared spectroscopy combined with intravascular ultrasound in carotid arteries. Int J Cardiovasc Imaging 32: 181-188, 2016

27) Ishikawa T, Hatakeyama K, Imamura T, et al.: Involvement of C-reactive protein obtained by directional coronary atherectomy in plaque instability and developing restenosis in patients with stable or unstable angina pectoris. Am J Cardiol 91: 287-292, 2003

28) Hansson GK: Inflammation, atherosclerosis, and coronary artery disease. N Engl J Med 352: 1685-1695, 2005

29) Nishi K, Itabe H, Uno M, et al.: Oxidized LDL in carotid plaques and plasma associates with plaque instability. Arterioscler Thromb Vasc Biol 22: 1649-1654, 2002

30) Pu J, Mintz GS, Brilakis ES, et al.: In vivo characterization of coronary plaques: novel findings from comparing greyscale and virtual histology intravascular ultrasound and near-infrared spectroscopy. Eur Heart $J$ 33: 372-383, 2012

31) Hornung M, Bertog SC, Franke J, Id D, Grunwald I, Sievert H: Evaluation of proximal protection devices during carotid artery stenting as the first choice for embolic protection. EuroIntervention 10: 1362-1367, 2015

32) Schofer J, Musiałek P, Bijuklic K, et al.: A prospective, multicenter study of a novel mesh-covered carotid stent: the CGuard CARENET trial (Carotid embolic protection using MicroNet). JACC Cardiovasc Interv 8: 1229-1234, 2015

33) Sato Y, Ogasawara K, Narumi S, et al.: Optimal MR plaque imaging for cervical carotid artery stenosis in predicting the development of microembolic signals during exposure of carotid arteries in endarterectomy: comparison of 4 T1-weighted imaging techniques. AJNR Am J Neuroradiol 37: 1146-1154, 2016

Corresponding author: Ichiro Nakagawa, MD, PhD, Departments of Neurosurgery, Nara Medical University, Shijo-cho 840, Kashihara, Nara 634-8521, Japan. e-mail: nakagawa@naramed-u.ac.jp. 